Stefano SCALERCIO* - Giuseppe LUZZi* ${ }^{*}$ - Michele LaUdati**

\title{
Nuovi reperti per la fauna microlepidotterologica degli ambienti forestali del Parco Nazionale della Sila, area MAB Unesco \\ (Lepidoptera Yponomeutoidea, Gelechioidea)
}

\begin{abstract}
Riassunto: Vengono segnalate per la prima volta nel territorio del Parco Nazionale della Sila 10 specie di microlepidoptera sottolineando il loro interesse faunistico per l'Italia meridionale. Ethmia pusiella è segnalata per la prima volta in Calabria. Cedestis subfasciella, Ypsolopha ustella, Schiffermuelleria schaefferella, Herrichia excelsella, Harpella forficella e Mirificarma cytisella sono segnalate per la prima volta in Italia meridionale. Argyresthia goedartella, A. pruniella e Sophronia sicariellus sono segnalate per la prima volta in Italia centro-meridionale.

Abstract: New records of microlepidoptera in forest habitats of the Sila National Park, MAB Unesco area (Lepidoptera Yponomeutoidea, Gelechioidea). Ten species of microlepidoptera are reported for the first time from the Sila National Park and their faunistic interest for southern Italy is pointed out. Ethmia pusiella is reported as new for the fauna of Calabria. Cedestis subfasciella, Ypsolopha ustella, Schiffermuelleria schaefferella, Herrichia excelsella, Harpella forficella and Mirificarma cytisella are reported as new for the fauna of southern Italy. Argyresthia goedartella, A. pruniella and Sophronia sicariellus are reported as new for the fauna of peninsular Italy.
\end{abstract}

Key words: Microlepidoptera, Fauna, Biodiversity, Calabria.

\section{INTRODUZIONE}

Il territorio del Parco Nazionale della Sila è stato oggetto di diverse ricerche dedicate allo studio della fauna lepidotterologica che hanno messo in evidenza una elevata concentrazione di biodiversità in questo territorio ricco di elementi relitti come il Nymphalidae Brenthis ino (Rottemburg, 1775) e di endemismi propri dell'Altopiano Silano come il Geometridae Itame messapiaria Sohn-Rethel, 1929 e lo Scythrididae Scythris acarioides Bengtsson, 1997.

Mentre la fauna dei macrolepidotteri diurni (ad es.: Scalercio, 2002, 2014a; Parenzan \& Porcelli, 2007) e notturni (ad es.: Parenzan \& Porcelli, 2007; Scalercio et al., 2008; Scalercio, 2014b) comincia a definirsi nei suoi lineamenti fondamentali, la fauna microlepidotterologica è pressoché ignota in quanto sono conosciute solo singole e isolate segnalazioni di pochissime specie e mancano del tutto studi specifici. Alcune specie vengono citate in lavori di ampio respiro come lo Scythrididae Scythris villari Agenjo, 1971 segnalato per Lorica da Bengtsson (1997), l'Eriocottidae Eriocottis nicola- eella Gibeaux, 1983 segnalato per Lorica da Parenti (2000) e il Coleophoridae Coleophora pseudorepentis Toll, 1960 segnalato per Lorica da Baldizzone et al. (1995). Altre specie sono ricordate in lavori dedicati a collezioni storiche come i Tortricidae Agapeta zoegana (Linnaeus, 1767) e Cochylis hybridella (Hübner, 1813) segnalati entrambe per Camigliatello da Trematerra (1992). Segnalazioni occasionali sono presenti anche in lavori dedicati ad altre aree geografiche come il Tortricidae Pelochrista mollitana (Zeller, 1847) segnalato per Sersale da Trematerra (1993), o in revisioni di un genere come è il caso del Tortricidae Clavigesta purdeyi (Durrant, 1911) segnalato per Longobucco da Larsen (2010).

Di particolare interesse sono la presenza del Crambidae Chrysocrambus brutiellus Bassi, 1985, descritto per la prima volta proprio su esemplari di Lorica (Bassi, 1985) e ora noto dell'Italia centro-meridionale (Bassi, 1998), e dello Scythrididae Scythris acarioides Bengtsson, 1997 anch'esso descritto su esemplari silani raccolti a Croce di Magara (Bengtsson, 1997) e a tutt'oggi noto solo per questo massiccio montuoso.

\footnotetext{
"Stefano Scalercio, Consiglio per la Ricerca in Agricoltura e l'Analisi dell'Economia Agraria, Unità di Ricerca per la Selvicoltura in Ambiente Mediterraneo, Contrada Li Rocchi, 87036 Rende (CS), Italia. E-mail: stefano.scalercio@entecra.it

${ }^{* *}$ Giuseppe Luzzi, Michele Laudati, Ente Parco Nazionale della Sila, Via Nazionale, 87055 Lorica, San Giovanni in Fiore (CS), Italia. E-mail: info@parcosila.it
} 
Lo scopo di questo contributo è di incrementare le conoscenze sulla fauna microlepidotterologica di questo importante territorio segnalando solo le specie per le quali mancavano segnalazioni per il meridione o la cui presenza era nota per la Calabria.

\section{MATERIALI E METODI}

Le località indagate (Fig. 1) sono caratterizzate da una copertura forestale variabile, ma sono generalmente dominate da Pinus laricio Poiret a cui si associano specie di diverse latifoglie. Di seguito se ne fornisce una descrizione dettagliata:

a. Croce di Magara, Spezzano Sila (CS), 1390 m, $39^{\circ} 19^{\prime} 21^{\prime \prime} \mathrm{N}, 16^{\circ} 28^{\prime} 29^{\prime \prime} \mathrm{E}$. L'area è attraversata dal corso del Fiume Neto ed è caratterizzata da piccole radure occasionalmente pascolate delimitate a monte da un bosco a Pinus laricio e a valle da un bosco ripario a Populus spp., Salix spp. e Alnus glutinosa (L.) Gaertn.;

b. Fossiata, Longobucco (CS), $1300 \mathrm{~m}, 39^{\circ} 23^{\prime} 43^{\prime}$ 'N, $16^{\circ} 35^{\prime} 55^{\prime}$ 'E. L'area è attraversata dal corso del Fiume Cecita lungo il quale si sviluppa un bosco ripario discontinuo ad Alnus glutinosa. Il paesaggio è dominato da boschi di Pinus laricio;

c. Lago Cecita, Spezzano della Sila (CS), 1163 m, $39^{\circ} 23^{\prime} 12^{\prime}$ N, $16^{\circ} 33^{\prime} 05^{\prime}$ 'E. La località di raccolta è situata ai margini del lago presso cui si sviluppa una formazione riparia a Salix spp. con sporadici esemplari di Alnus glutinosa che si continua sui versanti con una pineta a Pinus laricio;

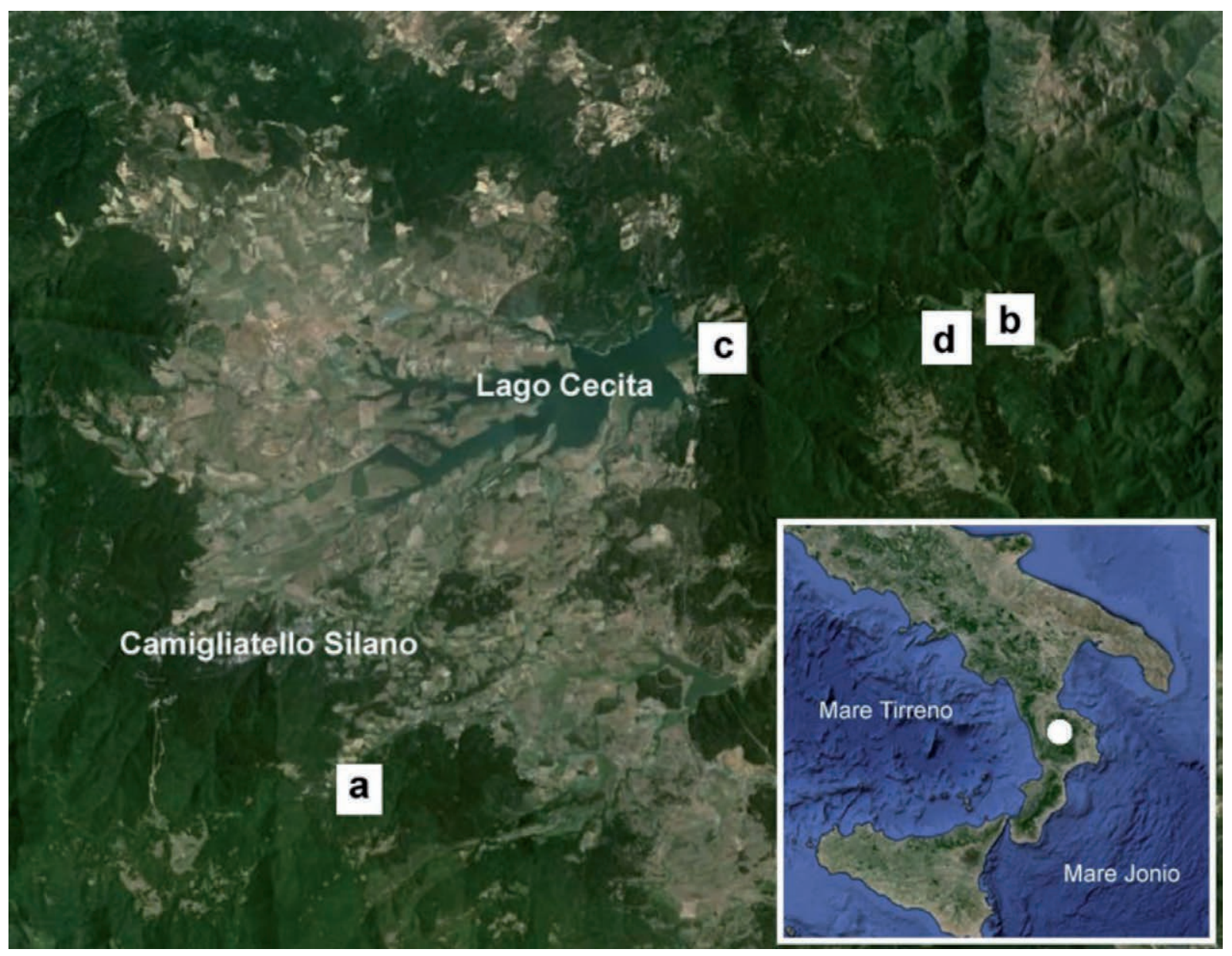

Fig. 1. Localizzazione dei siti di raccolta. a - Croce di Magara; b - Fossiata; c - Lago Cecita; d - Vivaio Sbanditi. 
d. Vivaio Sbanditi, Longobucco (CS), $1350 \mathrm{~m}$, $39^{\circ} 23^{\prime} 20^{\prime} \mathrm{N} ; 6^{\circ} 36^{\prime} 08^{\prime} \mathrm{E}$. Bosco misto di conifere la cui presenza può essere di origine naturale (Pinus laricio e Abies alba Mill.) o dovuta a rimboschimenti (Picea abies (L.) H.Karst. e Larix decidua Mill.). Sono presenti anche alcune latifoglie come Alnus glutinosa, Castanea sativa Mill. e Fagus sylvatica L.

Le raccolte sono state effettuate tramite sfalcio della vegetazione o cattura al volo durante le ore diurne e tramite l'utilizzo di una fonte luminosa durante le ore notturne. In particolare, presso il Vivaio Sbanditi è stata installata una trappola luminosa fissa tipo Rothamsted che viene attivata una volta a settimana.

Le specie sono state identificate dal primo autore con l'utilizzo di bibliografia specializzata (Parenti, 2000: diverse famiglie; Tokár et al., 2005: Oecophoridae; Huemer \& Karsholt, 1999: Gelechiidae) e dal dott. Giorgio Baldizzone con il confronto degli esemplari con il materiale presente nella propria collezione. Gli esemplari sono conservati presso la collezione dell'Unità di Ricerca per la Selvicoltura in Ambiente Mediterraneo.

\section{ELENCO DELLE SPECIE}

\section{Yponomeutoidea \\ Hyponomeutidae}

Cedestis subfasciella (Stephens, 1834) (Fig. 2)

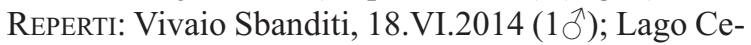
cita, 13.VIII.2013 (1ㅇ) (det. Giorgio Baldizzone), 13.VIII.2014 (1ふ) (det. Giorgio Baldizzone), 2.IX.2013 (1 $\left.\delta^{\Uparrow}\right)$ (det. Giorgio Baldizzone).

Specie nota in molti paesi europei (Karsholt et al., 2013), in Italia è conosciuta per il settentrione (Baraniak et al., 1995) e per il Lazio (Pinzari et al., 2010). La larva si sviluppa negli aghi di Pinus spp. e Abies spp. (Baldizzone et al., 2013). Gli esemplari sono stati raccolti di notte con trappole luminose.

Prima segnalazione per l'Italia meridionale.

\section{Argyresthiidae}

Argyresthia goedartella (Linnaeus, 1758) (Fig. 3)

REPERTI: Lago Cecita, 30.VII.2013 (1 9 ).

Specie nota in quasi tutta Europa (Karsholt et al., 2013), in Italia è conosciuta solo per il settentrione (Baraniak et al., 1995). La larva si sviluppa su Betula spp. e Alnus spp. (Baldizzone et al., 2013). L'esem- plare è stato raccolto di notte con trappole luminose. Prima segnalazione per l'Italia centro-meridionale.

Argyresthia pruniella (Clerck, 1759) (Fig. 4)

REPERTI: Lago Cecita, 30.VII.2013 (1우 2ð), 2.IX.2013 (1ठ).

Specie ampiamente diffusa in Europa (Karsholt et al., 2013), conosciuta in Italia per settentrione, Sicilia e Sardegna (Baraniak et al., 1995). La larva si sviluppa su fiori e giovani frutti di Cerasus spp., Corylus avellana L., Crataegus spp., Malus spp., Prunus spp., Sorbus spp. (Baldizzone et al., 2013). Gli esemplari sono stati raccolti di notte con trappole luminose.

Prima segnalazione per l'Italia centro-meridionale.

\section{Ypsolophidae}

Ypsolopha ustella (Clerck, 1759) (Fig. 5)

REPERTI: Vivaio Sbanditi, 27.X.2014 (1 $\overbrace{}^{\lambda})$.

Specie largamente diffusa in Europa (Karsholt et al., 2013), conosciuta in Italia per settentrione, Sicilia (Baraniak et al., 1995), Sardegna (Parenti, 2000) e Lazio (Pinzari et al., 2010). La larva si sviluppa su Quercus spp. (Baldizzone et al., 2013). L'esemplare è stato raccolto di notte con trappole luminose.

Prima segnalazione per l'Italia meridionale.

\section{Gelechioidea \\ Elachistidae}

Ethmia pusiella (Linnaeus, 1758) (Fig. 6)

REPERTI: Vivaio Sbanditi, 24.VI.2014 (10ิ, 1ㅇ), 26.VI.2014 (1 +), 13.VIII.2014 (1)

Specie nota in quasi tutta Europa (Karsholt et al., 2013), in Italia è conosciuta solo per il settentrione (Baldizzone et al., 1995) e la Basilicata (Parenti, 2000). La larva si sviluppa sulle Borraginacee Lithospermum officinale L. e Pulmonaria officinalis L. L'esemplare è stato raccolto di notte con trappole luminose.

Prima segnalazione per la Calabria.

\section{Oecophoridae}

Schiffermuelleria schaefferella (Linnaeus, 1758) (Fig. 7)

REPERTI: Vivaio Sbanditi, 18.VI.2014 (1ठ)

Specie distribuita in buona parte d'Europa (Karsholt et al., 2013), nota in Italia per settentrione (Baldizzone et al., 1995) e Lazio (Pinzari et al., 2010). La larva si 

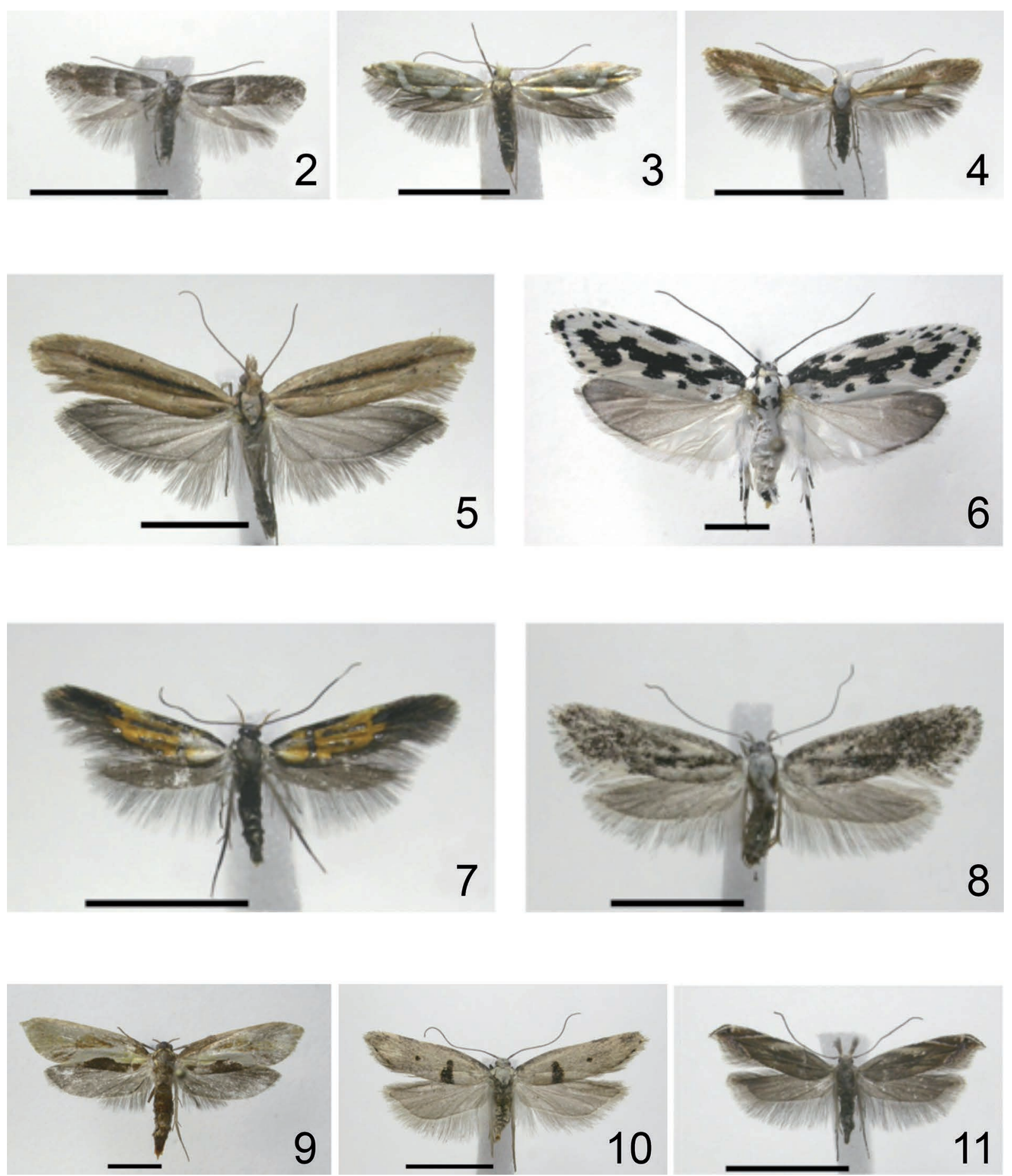

Fig. 2-11. Raffigurazione delle specie trattate; 2 - Cedestis subfasciella, Lago Cecita, 13.VIII.2013; 3 - Argyresthia goedartella, Lago Cecita, 30.VII.2013; 4 - Argyresthia pruniella, Lago Cecita, 30.VII.2013; 5 - Ypsolopha ustella, Vivaio Sbanditi, 27.X.2014; 6 - Ethmia pusiella, Vivaio Sbanditi, 26.VI.2014; 7 - Schiffermuelleria schaefferella, Vivaio Sbanditi, 18.VI.2014; 8 - Herrichia excelsella, Lago Cecita, 13.VIII.2014; 9 - Harpella forficella, Lago Cecita, 30.VII.2013; 10 - Mirificarma cytisella, Vivaio Sbanditi, 26.VII.2014; 11 - Sophronia sicariellus, Croce di Magara, 8.VII.2014. Lunghezza della barra: 5 mm. 
sviluppa su legno morto di Salicacee, Fagacee e Rosacee (Parenti, 2000). L'esemplare è stato catturato in volo al crepuscolo con un retino.

Prima segnalazione per l'Italia meridionale.

Herrichia excelsella Staudinger, 1871 (Fig. 8) REPERTI: Lago Cecita, 30.VII.2013 (1 $\left.{ }^{\Uparrow}\right)$ (det. Giorgio Baldizzone), 13.VIII.2013 (1 + ) (det. Giorgio Baldizzone), 13.VIII.2014 (4웅); Fossiata, 13.VIII.2013 (1ㅇ) (det. Giorgio Baldizzone); Vivaio Sbanditi, 5.VIII.2014 (2仓ð)

Specie conosciuta per Austria, Svizzera, Germania, Portogallo, Spagna, Francia (Karsholt et al., 2013) e in Italia per Trentino-Alto Adige, Piemonte, Valle d'Aosta, Lazio e Sicilia (Baldizzone et al., 2013). La biologia larvale è ignota, ma si suppone che le larve si nutrano di legno morto di Pinus spp. e altre conifere (Baldizzone et al., 2013). Gli esemplari sono stati raccolti di notte con trappole luminose.

Prima segnalazione per l'Italia meridionale.

Harpella forficella (Scopoli, 1763) (Fig. 9) REPERTI: Lago Cecita, 30.VII.2013 (2o+ $)$ ), 13.VIII. $2013(1$ ㅇ)

Specie largamente diffusa in Europa ad eccezione delle Isole Britanniche (Karsholt et al., 2013), nota in Italia per settentrione (Baldizzone et al., 1995) e Lazio (Pinzari et al., 2010). Le larve si nutrono di legno morto (Baldizzone et al., 2013). Gli esemplari sono stati raccolti di notte con trappole luminose.

Prima segnalazione per l'Italia meridionale.

\section{Gelechiidae}

Mirificarma cytisella (Treitschke, 1833) (Fig. 10)

REPERTI: Vivaio Sbanditi, 18.VI.2014 (1へ), 26.VI.2014 (2웅), 26.VII.2014 (1우)

Specie diffusa in Europa centrale e meridionale (Karsholt et al., 2013), nota in Italia per settentrione (Baldizzone et al., 1995) e Lazio (Pinzari et al., 2010). Le larve si sviluppano su diverse ginestre e Ononis spi- nosa (Baldizzone et al., 2013). Gli esemplari sono stati raccolti di notte con trappole luminose.

Prima segnalazione per l'Italia meridionale.

Sophronia sicariellus (Zeller, 1839) (Fig. 11)

REPERTI: Croce di Magara, 8.VII.2014 (1 $\overbrace{}^{\Uparrow})$

In Europa è presente con discontinuità in molti paesi ma con maggiore frequenza in Europa centrale (Karsholt et al., 2013), nota in Italia solo per il settentrione (Baldizzone et al., 1995). Le larve si sviluppano su Achillea spp., Artemisia campestris L., Inula oculuschristi L. (Baldizzone et al., 2013). L'esemplare è stato catturato di giorno tramite sfalcio della vegetazione erbacea.

Prima segnalazione per l'Italia centro-meridionale.

\section{CONCLUSIONI}

Questo lavoro modifica le conoscenze sull'areale di 10 specie che si amplia includendo il Sud della penisola Italiana. Inoltre conferma l'elevata biodiversità degli ambienti forestali del Parco Nazionale della Sila. Molte delle specie segnalate sono strettamente legate da rapporti trofici con la copertura forestale. In particolare il rinvenimento degli Oecophoridae Schiffermuelleria schaefferella, Harpella forficella e Herrichia excelsella, le cui larve si nutrono di legno morto o marcescente, hanno una particolare importanza da un punto di vista ecologico. Alcune specie di questa famiglia potrebbero essere utilizzate in futuro come indicatori delle foreste vetuste caratterizzate, tra l'altro, da una buona presenza di legno morto.

\section{RINGRAZIAMENTI}

Si ringrazia Giorgio Baldizzone, Asti, per la determinazione di alcune specie. Lavoro parzialmente finanziato dall'Ente Parco Nazionale della Sila attraverso la Convenzione fra lo stesso Ente e l'Unità di Ricerca per la Selvicoltura in Ambiente Mediterraneo denominata "Progetto di monitoraggio dei lepidotteri notturni attraverso l'utilizzo di trappole luminose tipo Rothamsted". 
BiBliogRAFIA

Baldizzone G., Gozmány L., Huemer P., Karsholt O., Lvovsky A., Parenti U., Passerin d’Entrèves P., Riedl T., Varalda P.G., Zangheri S., 1995 - Lepidoptera Gelechioidea. In: Minelli A., Ruffo S., La Posta S. (eds.), Checklist delle Specie della Fauna Italiana, 83, Calderini, Bologna.

Baldizzone G., Cabella C., Fiori F., Varalda P.G., 2013 - I Lepidotteri del Parco Naturale di Marcarolo. Memorie dell'Associazione Naturalistica Piemontese, XII, 349 pp.

Baraniak E., Gaedike R., Karsholt O., Triberti P., Zangheri S., 1995 - Lepidoptera Tineoidea II. In: Minelli A., Ruffo S., La Posta S. (eds.), Checklist delle Specie della Fauna Italiana, 82, Calderini, Bologna.

BASsi G., 1985 - Contributo allo studio delle Crambinae (Lepidoptera, Pyralidae) I: Specie mediterranee nuove o interessanti. Bollettino del Museo Regionale di Scienze Naturali di Torino, 3(1): 73-78.

BASSI G., 1998 - Segnalazioni faunistiche italiane: 366. Bollettino Società entomologica italiana, 130 (3): 277-278.

Bengtsson B.A., 1997 - Microlepidoptera of Europe. Volume 2. Scythrididae. Apollo Books, Stenstrup, 301 pp.

Karsholt O., Nieukerken E.J. van, DE Jong Y.S.D.M., 2013. Lepidoptera, Moths. Fauna Europaea version 2.6, http://www.faunaeur.org

LARSEN K., 2010 - The genus Clavigesta (Lepidoptera: Tortricidae) with description of two new species. Phegea, 38(2): 41-54.

Parenti U., 2000 - A guide to the Microlepidoptera of Europe. Guide I. Museo Regionale di Scienze Naturali Torino, 426 pp.

Parenzan P., Porcelli F., 2007 - I macrolepidotteri italiani. Fauna Lepidopterorum Italiae (Macrolepidoptera). Phytophaga, 15(2005-2006). Allegato in pdf: 1-1051.

PinZARi M., PInZARi M., Zilli A., 2010 - Deep lepidopterological exploration of Mt Cagno and surroundings (Central Italy), a restricted mountain massif and hotspot for butterfly and moth diversity. Bollettino dell'Associazione Romana di Entomologia, 65(1-4): 3-383.

SCALERCio S., 2002 - La fauna a Lepidotteri Ropaloceri della Sila Greca (Italia meridionale) (Lepidoptera Hesperioidea e Papilionoidea). Memorie della Società Entomologica Italiana, 81: 167-204.

SCALERCiO S., 2014a - New distributional data of butterflies in the middle of the Mediterranean Basin, an area very sensitive to expected climate change. Dataset Paper in Science, 2014: 8 pp., 5 dataset items.

SCALERCIO S., 2014b - Nuovi dati di distribuzione dei macrolepidotteri eteroceri della fauna calabrese (Insecta Lepidoptera). Memorie della Società entomologica Italiana, 90(1): 3-59.

SCAlercio S., Infusino M., TusCAno J., 2008 - I Macrolepidotteri notturni della faggeta di Monte Curcio, Sila Grande (Calabria, Italia meridionale) (Lepidoptera). Quaderni della Stazione di Ecologia, Civico Museo di Storia Naturale di Ferrara, 18 : 5-19.

TokÁr Z., Lvovsky A., Huemer P., 2005 - Die Oecophoridae s.l. (Lepidoptera) Mitteleuropas. František Slamka, Bratislava, Slovakia, $120 \mathrm{pp}$.

Trematerra P., 1992 - I Cochylini della collezione A. Fiori nel museo di Storia Naturale di Milano. Memorie della Società entomologica Italiana, 70(2) (1991): 267-285.

Trematerra P., 1993 - Un piccolo contributo alle conoscenze dei tortricidi dell'isola di Capraia (Arcipelago Toscano). Rivista del Museo Civico di Scienze Naturali “E. Caffi”, Bergamo, 16: 149-152. 